\title{
Design of Miniaturized Narrowband Absorbers Based on Resonant-Magnetic Inclusions
}

\author{
Filiberto Bilotti, Senior Member, IEEE, Alessandro Toscano, Kamil Boratay Alici, Ekmel Ozbay, and Lucio Vegni
}

\begin{abstract}
In this paper, we present the design of miniaturized narrowband-microwave absorbers based on different kinds of magnetic inclusions. The operation of the proposed components originates from the resonance of a planar array of inclusions excited by an incoming wave with a given polarization. As in common absorber layouts, a $377 \Omega$ resistive sheet is also used to absorb the electromagnetic energy of the impinging field. Since the planar array of magnetic inclusions behaves at its resonance as a perfect magnetic conductor, the resistive sheet is placed in close proximity of the resonating inclusions, without perturbing their resonance condition. In contrast to other typical absorber configurations presented in the literature, the absorber proposed in this paper is not backed by a metallic plate. This feature may be useful for stealth applications, as discussed thoroughly in the paper. The other interesting characteristic of the proposed absorbers is the subwavelength thickness, which has shown to depend only on the geometry of the basic resonant inclusions employed. At first, regular split-ring resonators (SSRs) disposed in an array configuration are considered and some application examples are presented. Absorbers based on SRRs are shown to reach thickness of the order of $\lambda_{0} / 20$. In order to further squeeze the electrical thickness of the absorbers, multiple SRRs and spiral resonators are also used. The employment of such inclusions leads to the design of extremely thin microwave absorbers, whose thickness may even be close to $\lambda_{0} / 100$. Finally, some examples of miniaturized absorbers suitable for a practical realization are proposed.
\end{abstract}

Index Terms-Metamaterials, microwave absorbers, miniaturized magnetic inclusions, split-ring resonators.

\section{INTRODUCTION}

$\mathbf{T}$ HE NEED for microwave absorbers and radar-absorbing materials is ever growing in different aspects of ongoing technologies. In particular, absorbers play a key role in military applications and are mainly employed for the reduction of the radar signature of aircraft, ships, tanks, and other targets. Apart from stealth and camouflage applications, microwave absorbers are also used in both military and civil applications to reduce the electromagnetic interference among microwave components and/or electronic circuits mounted on the same platform. The employment of microwave absorbers, enabling the absorption of the reflected and surface waves, is also needed to improve the

Manuscript received May 7, 2009; revised January 15, 2010; accepted May 13, 2010. Date of publication June 21, 2010; date of current version February 16, 2011.

F. Bilotti, A. Toscano, and L. Vegni are with the Department of Applied Electronics, University of Roma Tre, Rome 00146, Italy (e-mail: bilotti@uniroma3.it; toscano@ieee.org; vegni@uniroma3.it).

K. B. Alici and E. Ozbay are with the Nanotechnology Research Center, Bilkent University, Ankara 06800, Turkey (e-mail: bora@fen.bilkent.edu.tr; ozbay@bilkent.edu.tr).

Color versions of one or more of the figures in this paper are available online at http://ieeexplore.ieee.org.

Digital Object Identifier 10.1109/TEMC.2010.2051229 performance of both transmissive and radiating components. For instance, in antenna applications, absorbers may be employed to reduce the back-radiation of microstrip radiators. This is a key aspect in the design of radiators for either high-precision systems (e.g., antennas for earth-stations of satellite-navigation systems) or mobile communications (e.g., antennas for mobilephone terminals with reduced radiation towards the user head).

Whatever the application for which the absorber is intended, the reduction of its electrical thickness is always one of the challenging aspects. Other important issues are related to the polarization dependence, angular bandwidth, and operating bandwidth.

Significant advancements in absorber technology have been recently obtained through the employment of artificial electromagnetic materials at different frequencies, spanning from microwaves to tetrahertz $(\mathrm{THz})$, and optical frequencies. Some of the latest results can be found in [1]-[4], and reference therein.

The absorbers employed in everyday applications are usually backed by a metallic plate [5]-[10]. The metallic backing plays two main roles. On one hand, it is used to avoid power transmission on the other side of the absorber. On the other hand, it enables a boundary condition useful to create a reflected component that, combined with the impinging wave, cancels out the reflection from the screen. This phenomenon is well evident in the principle of operation of the Salisbury screen [5], [6], the simplest microwave absorber, but it is anyway present in all the layouts involving a metallic backing. In the Salisbury screen layout, a $377 \Omega$ resistive sheet is placed a quarter-wavelength apart from a metallic plate, where the tangential component of the electric field has its maximum amplitude. ${ }^{1}$

As far as resonant absorbers are concerned (i.e., absorbers that cancel the reflection in a narrow frequency band), the presence of the metallic plate may represent a problem, especially, for stealth applications. If we want to hide an object made by a certain material at a given frequency, in fact, the employment of conventional absorbers with a metallic backing makes the material object a metallic object and, thus, at frequencies other than the one for which the absorbing structure has been designed, the radar cross section (RCS) of the object may increase.

In order to avoid a metallic backing, proper resonant structures may be employed. In this frame, it is possible to make use of metamaterials that are artificial materials exhibiting properties not readily found in natural materials [11]. Such manmade materials consist of microwave frequencies of metallic

\footnotetext{
${ }^{1}$ Since the section where the resistive sheet is placed is $\lambda / 4$ apart from the metallic plate, that section is characterized by the boundary condition of a perfect magnetic conductor (PMC), where the tangential component of the electric field has its maximum.
} 
resonant inclusions immersed in a host dielectric. Since the dimension of and the separation between the inclusions are both very small compared to the operating wavelength, it is usually possible to model the macroscopic behavior of the composite material in terms of the effective permittivity and permeability [12]. Due to the resonance behavior of the inclusions, also the effective permittivity and permeability exhibit a dispersive behavior characterized by a resonance and, if the inclusions are properly designed, it is also possible to obtain negative values for the effective constitutive parameters. Moreover, metamaterials usually exhibit high losses around the resonance frequency and this aspect sometimes limits their employment in practical components requiring high efficiencies. However, as far as absorbers are concerned, losses may help to absorb the impinging electromagnetic power. The inherent-resonance behavior of metamaterials and their lossy nature, thus, make such materials suitable candidates for the design of resonant-microwave absorbers without a metallic backing.

Recently, some of the authors have presented theoretical investigations relating to the design of compact microwave absorbers, made by a proper combination of two metamaterial slabs [13]-[16]. The operation principle of that layout is based on the anomalous surface resonance, arising at the interface between two slabs, characterized by oppositely signed values of the real parts of permittivities and/or permeabilities. ${ }^{2}$ From the theoretical point of view, in fact, it may be readily verified that these bilayers, when excited by an impinging electromagnetic wave, may support a localized interface resonance, whatever the total thickness of the entire setup is, for any angle of incidence, and whatever the nature of the backing on the other side of the structure. Since the amplitude of the field is huge at the interface between the two metamaterial slabs and the resonance is basically confined at the interface, a resistive sheet is placed right at the interface between the two slabs, absorbing, thus, most of the power of the impinging wave. The practical implementation of this layout involves the realization of both the slab with the negative real part of the permittivity and the slab with the negative real part of the permeability. For the former slab, electric inclusions (e.g., wire inclusions [17]) are to be used, while, for the latter slab, magnetic inclusions (e.g., split ring resonators (SRRs) [18] at the lower microwave frequencies) are needed.

Since from the practical point of view, it would be rather difficult to employ two sets of resonant inclusions and make the two-paired slabs resonating in a proper way [16], Bilotti et al. were stimulated to find an easier layout, based on a different resonant phenomenon and employing only a single set of inclusions.

The results of these investigations conducted at lower microwave frequencies, are presented in this paper. In Section II, a brief motivation of the choice of the resonant inclusions of the magnetic kind is given. Then, different magnetic inclusions are considered to verify the concept of resonant miniaturized microwave absorbers without a metallic backing. At first, in

\footnotetext{
${ }^{2}$ In [13]-[16], we have ideally modeled the two metamaterials as homogeneous and isotropic materials, characterized by effective permittivity and permeability both described by Lorentz dispersion.
}

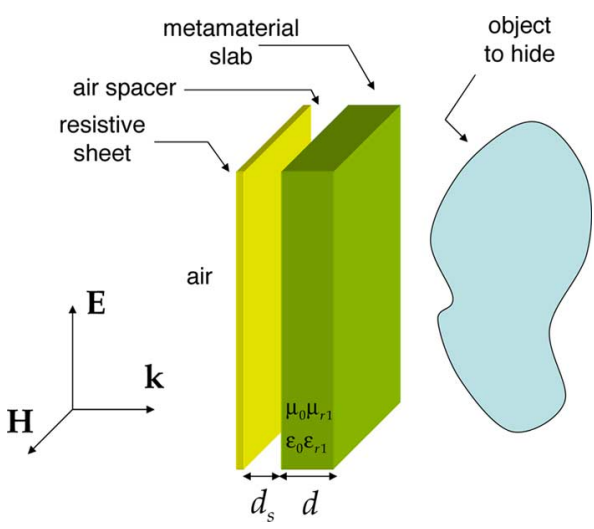

Fig. 1. Sketch describing the structure of the proposed resonant absorber made by a resistive sheet and a metamaterial slab.

Section III, we consider the employment of regular SRRs, then, in order to achieve a further reduction of the absorber thickness, the use of multiple SRRs (MSRRs) and spiral resonators (SRs) is proposed in Sections IV and V, respectively.

\section{CHOICE OF THE RESONANT INCLUSIONS}

In order to save space and reduce the electrical thickness of the absorbers, it is advisable to employ an artificial medium behaving, at the desired frequencies, as an artificial magnetic conductor (AMC). As already pointed out in some recently presented layouts of absorbers based on the employment of frequency selective surfaces and high impedance surfaces, in fact, due to the boundary condition imposed by the AMC, the resistive sheet can be placed very close to the AMC itself [7]-[9]. However, in the aforementioned layouts, the resonance behavior, leading to the AMC boundary condition, is always obtained through the help of a metallic plate, backing the structure of the absorber.

If we want to avoid a metallic backing, we may refer to the sketch shown in Fig. 1. A resistive sheet is placed at a certain distance $d_{s}$ apart from a slab of thickness $d$, described by the constitutive parameters of a linear, isotropic, and homogeneous material $\left(\varepsilon_{1}=\varepsilon_{0} \varepsilon_{r 1}, \mu_{1}=\mu_{0} \mu_{r 1}\right)$ and there is no metallic plate between the absorber and the whole scenario on the right-hand side (objects to hide, etc.)

For the sake of simplicity, let us consider the structure excited by a plane wave normally impinging from the left-hand side. Applying the equivalent transmission-line representation as shown in Fig. 2 and considering the two thicknesses $d$ and $d_{s}$ very small compared to the operating wavelength, the reflection coefficient at the input interface is given by, as shown (1), at the bottom of the next page, where $R$ is the total resistance associated to the resistive sheet and $Z_{L}$ is the load impedance describing a generic scenario behind the absorber.

In order to verify what kind of inclusion is more suitable to obtain at a given frequency, a zero-reflection coefficient in the case of subwavelength dimensions, we just evaluate the limit of the expression in (1) when $\varepsilon_{r 1} \rightarrow \pm \infty$ (this is the ideal case of resonant inclusions of the electric kind) and $\mu_{r 1} \rightarrow \pm \infty$ (this 


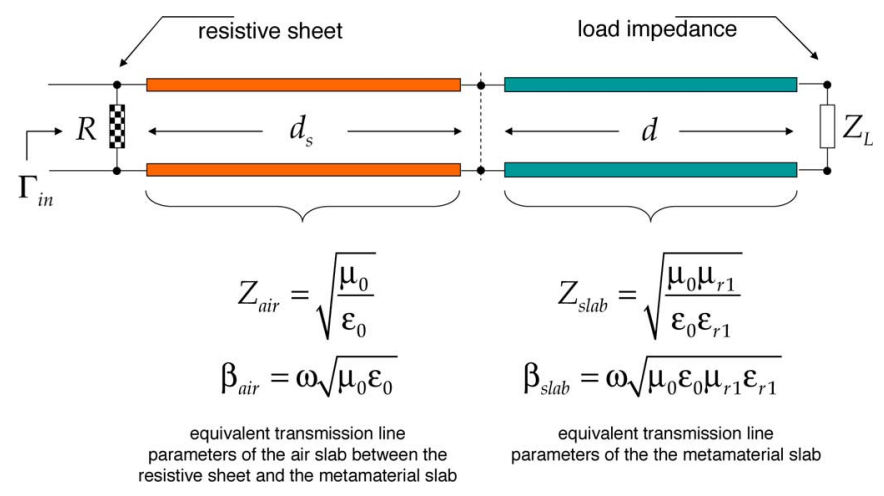

Fig. 2. Equivalent transmission-line representation of the metamaterial absorber described in Fig. 1.

is the ideal case of resonant inclusions of the magnetic kind). Calculating the two limits, we obtain

$$
\begin{aligned}
& \lim _{\varepsilon_{r 1} \rightarrow \infty} \Gamma_{\text {in }} \simeq-1 \\
& \lim _{\mu_{r 1} \rightarrow \infty} \Gamma_{\text {in }} \simeq \frac{R \sqrt{\varepsilon_{0}}-\sqrt{\mu_{0}}}{R \sqrt{\varepsilon_{0}}+\sqrt{\mu_{0}}} .
\end{aligned}
$$

As previously anticipated, provided that the resistive sheet is designed to offer a total resistance $R=\sqrt{\mu_{0} / \varepsilon_{0}}=120 \pi=$ $377 \Omega$ (the same as in regular Salisbury screen layouts), we may have a thin absorber by employing resonant inclusions of the magnetic kind, that will result in a PMC boundary condition, whatever the values of $d$ and $d_{s}$ are (under the hypothesis $k_{0} d, k_{0} d_{s} \ll 1$ ), for any loading impedance (i.e., whatever the scenario or the object on the other side of the absorber is), and for any value of $\varepsilon_{r 1}$.

Therefore, considering a wall made by a planar array of inclusions of the magnetic kind, such as the SRRs, if the impinging field is polarized in such a way so as to properly excite the inclusions, the reflected field at the resonance would be in-phase with the incident field and most of the impinging power would be absorbed in the resistive sheet. Some preliminary numerical results showing the effectiveness of the proposed approach have been briefly presented by some of the authors in [19], without giving details on the theoretical aspects and the device performance.

It is worth remarking here that the total electrical thickness of the absorber $d+d_{s}$ depends only on the electrical dimensions of the resonant inclusions employed. For this reason, in the following sections, we will propose some different designs, based not only on the SRRs, but also on other resonant inclusions that, while having the same dimensions of regular SRRs, exhibit, indeed, lower resonant frequencies.

\section{RESONANT ABSORBERS BASED ON SRRS}

In order to demonstrate the concept of subwavelengthmicrowave absorbers made by magnetic resonant inclusions

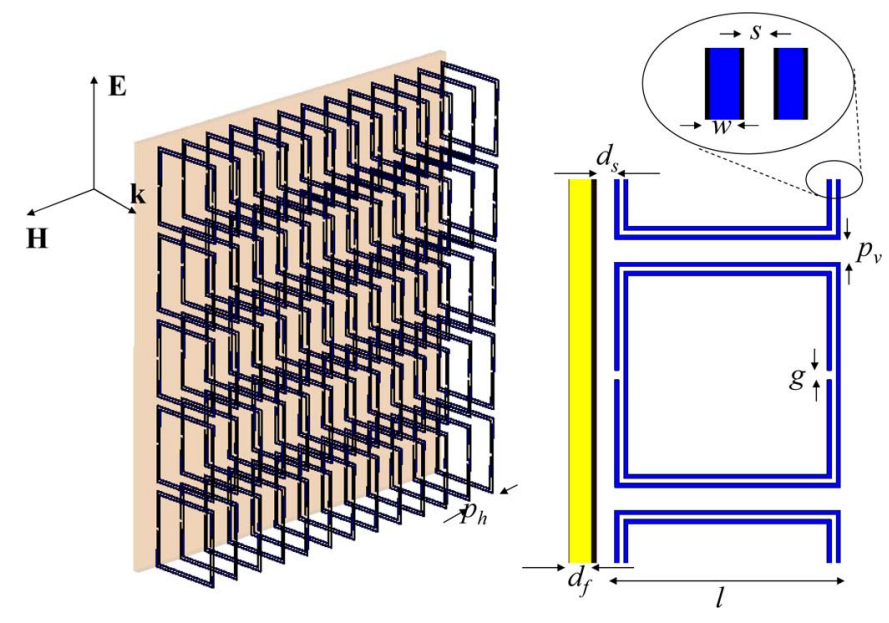

Fig. 3. Geometrical sketch of a microwave absorber based on SRR resonant magnetic inclusions. The absorber is made by a resistive film of thickness $d_{f}$ and a planar array of SRRs spaced $d_{s}$ from the resistive film.

without a metallic backing, we have numerically simulated the behavior of the structure depicted in Fig. 3. In this case, a planar array of regular air immersed SRRs ${ }^{3}$ is excited by a normally directed plane wave, having the magnetic field parallel to the axes of the SRRs.

This structure has been simulated employing CST Microwave Studio, a full-wave commercial code based on the finiteintegration technique [22]. The finite-planar array of SRRs has been placed inside a hollow waveguide with inhomogeneous boundaries: top and bottom walls are described by the boundary condition of a perfect electric conductor (PEC), left and right walls by the condition of a PMC. In this way, since the waveguide supports a TEM mode with a vertically polarized electric field and a horizontally polarized magnetic field, we were able to simulate a TEM plane wave directed along the waveguide axis, propagating in the waveguide, and normally impinging on the array of SRRs.

The geometrical dimensions of the SRRs are chosen [18] so as to return a resonance at approximately $2 \mathrm{GHz}$. The reflected and transmitted powers as a function of the frequency reported in Fig. 4 confirm the expected absorbing behavior.

In Fig. 5, the amplitude of the electric field along the vertical section of the waveguide near the SRRs at the resonant frequency is depicted. From this graph, it may be easily verified how the field is highly concentrated around the resonating SRRs, is absorbed in the resistive sheet, and is very low on the right-hand side of the structure, giving rise to a very low transmission. A time animation would show how the incident wave is actually a propagating wave with a negligible standing-wave contribution

\footnotetext{
${ }^{3}$ Absorbing properties of planar arrays of SRRs in C-shaped configuration have been previously studied in [20] and [21] for a different layout, characterized by a metallic backing.
}

$$
\Gamma_{\text {in }}=\frac{\left[j Z_{L}-\omega \mu_{0}\left(d+d_{s} \mu_{r 1}\right)\right]+R\left[j+\omega\left(d+d_{s} \mu_{r 1}\right) \sqrt{\mu_{0} \varepsilon_{0}}-Z_{L}\left(j \sqrt{\varepsilon_{0}}+\omega \varepsilon_{0}\left(d+d_{s} \varepsilon_{r 1}\right)\right]\right.}{\left[-j Z_{L}+\omega \mu_{0}\left(d+d_{s} \mu_{r 1}\right)\right]+R\left[-j+\omega\left(d+d_{s} \mu_{r 1}\right) \sqrt{\mu_{0} \varepsilon_{0}}+Z_{L}\left(-j \sqrt{\varepsilon_{0}}+\omega \varepsilon_{0}\left(d+d_{s} \varepsilon_{r 1}\right)\right]\right.}
$$




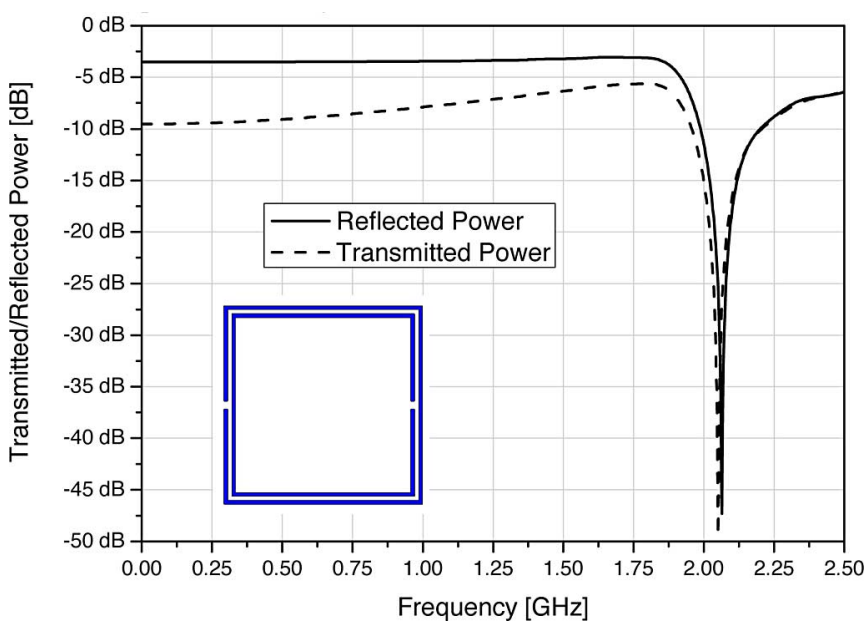

Fig. 4. Reflected and transmitted power from and through the absorber depicted in Fig. 3. The geometrical dimensions of the absorber are: $l=5 \mathrm{~mm}, w=$ $0.1 \mathrm{~mm}, s=0.1 \mathrm{~mm}, g=0.1 \mathrm{~mm}, p_{h}=2 \mathrm{~mm}, p_{v}=0.5 \mathrm{~mm}, d_{f}=0.5 \mathrm{~mm}$, and $d_{s}=0.5 \mathrm{~mm}$. The total resistance of the resistive sheet is $377 \Omega$.
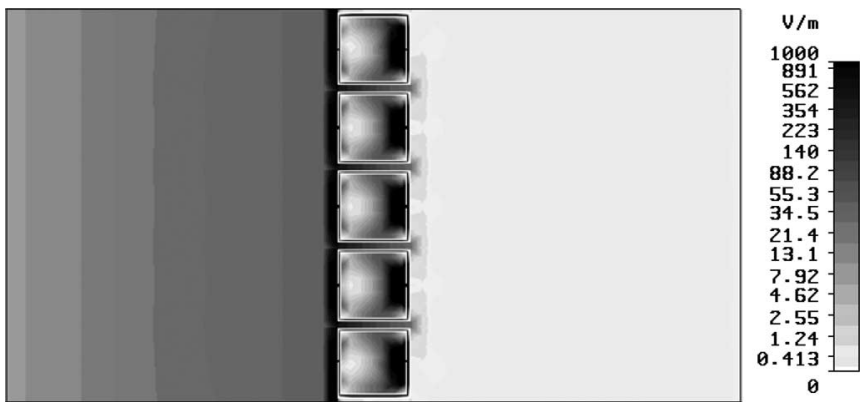

Fig. 5. Amplitude of the electric field along the vertical section of the waveguide near the SRRs at the resonant frequency $2.05 \mathrm{GHz}$. The geometrical dimensions of the absorber and the total resistance of the resistive sheet are the same as in the caption of Fig. 3 .

(i.e., the reflection coefficient at the left-hand side interface is very low).

At the resonant frequency $(2.05 \mathrm{GHz})$, the free-space wavelength is approximately $14.6 \mathrm{~cm}$. Since the SRR side $l$ is only $5 \mathrm{~mm}\left(\lambda_{0} / 29\right)$ long and the thicknesses of the air spacer and of the resistive sheet are $d_{s}=0.5 \mathrm{~mm}$ and $d_{f}=0.5 \mathrm{~mm}$, respectively, the total thickness of the absorber is only $6 \mathrm{~mm}$, i.e., around $\lambda_{0} / 24$.

In order to verify the expected independence of the absorbing features on the boundary condition at the right-hand side interface of the absorber (i.e., the independence on the load impedance $Z_{L}$ ), we have performed a simulation of a real-life scenario where the absorber is used to hide a metallic object (a sphere in this case) placed at different positions behind the screen (see Fig. 6). The radius of the sphere has been chosen in such a way that its RCS in the waveguide has its first sharp maximum ( $0 \mathrm{~dB}$ in the lossless case) around the resonant frequency of the SRRs $(2.05 \mathrm{GHz})$.

This peak, revealing in normal circumstances the presence of the sphere, is no longer present in the results shown in Fig. 6(a)-

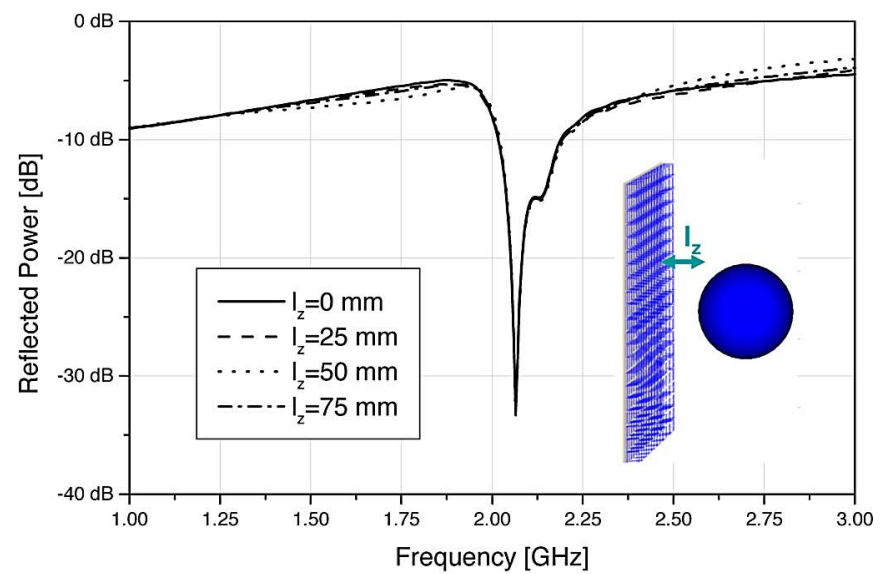

(a)



(b)

Fig. 6. (a) Reflected and (b) transmitted power from and through the SRR absorber used to hide a metallic sphere of radius $30 \mathrm{~mm}$. The position of the sphere $l_{z}$ is varied within a half-wavelength in order to verify the stability of the absorbing features for both inductive and capacitive loading impedances. When the electrical distance of the metallic object is very close to the absorber, the object mainly resembles an inductive lumped load. In contrast, when the electrical distance between the absorber and the metallic object excedes a quarter of the free-space wavelength, the object behaves rather as a capacitive load.

when the absorber is used--whatever the position of the sphere (i.e., for any loading impedance placed on the back interface).

In Fig. 7, we show the behavior of the absorber as a function of the incidence angle. The numerical simulation has been performed by placing the absorber inside a hollow waveguide with PEC walls.

In this way, increasing the horizontal dimension of the waveguide beyond the cutoff length, we may easily simulate the variation of the incidence angle of the impinging plane wave. ${ }^{4}$

${ }^{4}$ It is well known, in fact, that the fundamental $\mathrm{TE}_{10}$ mode of a hollow PEC rectangular waveguide can be represented as a superposition of two plane waves propagating with the angle

$$
\theta=\arccos \left[\sqrt{1-\frac{\pi^{2}}{k_{0}^{2} a^{2}}}\right]
$$

with respect to the axis of the waveguide, being $a$ the length of the horizontal side of the waveguide and $k_{0}$ the free-space wavenumber. At a given frequency, thus, the angle of incidence can be changed just varying the horizontal dimension $a$. 




(a)

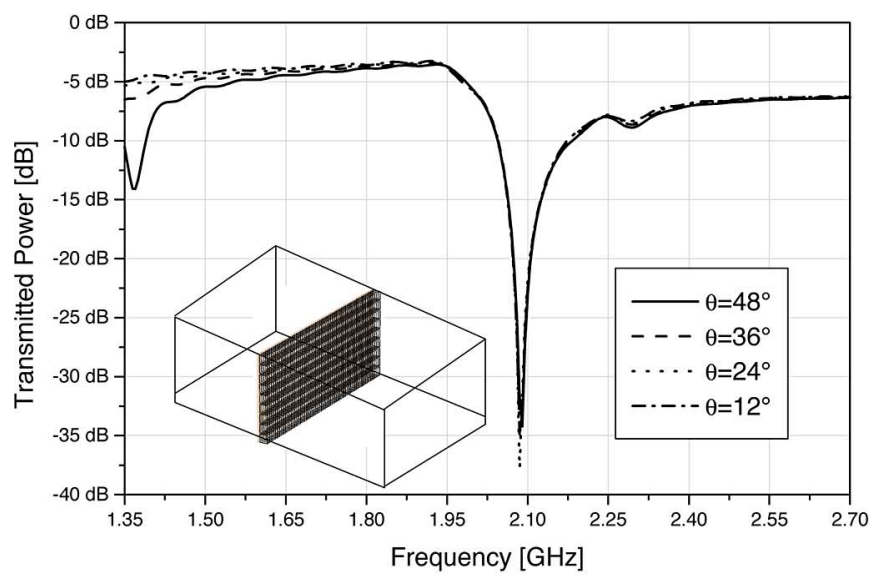

(b)

Fig. 7. (a) Reflected and (b) transmitted power from and through the SRR absorber placed within a waveguide with PEC walls. The horizontal dimension of the waveguide has been varied to change the angle of incidence.

For a deep physical explanation of the angular independent behavior of this and more general metamaterial structures, see [23].

\section{RESONANT ABSORBERS BASED ON MSRRS}

As previously remarked, the thickness of the proposed absorber depends mainly on the dimensions of the magnetic inclusions used. In order to obtain a further reduction of the absorber thickness, other kinds of resonating magnetic inclusions should be considered. In particular, the authors have recently proposed a new analytical model to describe through a parallel $L C$ equivalent circuit the electromagnetic behavior of both MSRRs and SRs that are magnetic inclusions capable of achieving improved miniaturization scales [24], [25].

MSRRs are a straightforward extension of the regular SRRs. They are obtained from the SRRs by just adding new split rings in the inner part of the inclusions. In this way, the new distributed capacitances (one for each added ring) between adjacent rings, all connected in parallel [24], [25], increase the total capacitance associated to the inclusion, thus, reducing the resonant frequency, while keeping unchanged the space occupancy of the magnetic inclusion.

Under the assumptions that all the mutual interactions between nonadjacent rings are negligible, the total capacitance and inductance of the squared MSRR are given by Bilotti et al. [24], [25]

$$
\begin{aligned}
C_{\mathrm{MSRR}} & =\frac{N-1}{2}[2 l-(2 N-1)(w+s)] C_{0} \\
L_{\mathrm{MSRR}} & =4 \mu_{0}[l-(N-1)(s+w)]\left[\ln \left(\frac{0.98}{\rho}\right)+1.84 \rho\right]
\end{aligned}
$$

where

$$
\begin{aligned}
\rho & =\frac{(N-1)(w+s)}{l-(N-1)(w+s)} \\
C_{0} & =\varepsilon_{0} \frac{K\left(\sqrt{1-k^{2}}\right)}{K(k)} \\
K(x) & =\int_{0}^{\pi / 2} \frac{1}{\sqrt{1-x \sin ^{2} \varphi}} d \varphi \quad k=\frac{s / 2}{w+s / 2}
\end{aligned}
$$

with $l$ being the length of the external ring, $w$ the width of the strip, $s$ the separation between two adjacent rings, and $N$ the number of the rings. Once all the geometrical parameters are known, (3) and (4) may be used to determine the resonant frequency of the MSRR as a function of the number $N$ of the rings. It is easily found that, increasing $N$ beyond a certain threshold, the resonant frequency does not reduce any more and that a saturation effect takes place [24], [25].

Here, we are interested in using such magnetic inclusions to obtain more miniaturized resonant absorber. The structure of the absorber is reported in Fig. 8, where all the relevant geometrical parameters are also displayed. In order to verify the reduction of the resonant frequency by using MSRRs instead of regular SRRs, the simulations are performed keeping unchanged all the geometrical parameters reported in the caption of Fig. 4.

The results of the power reflected from and transmitted through the absorber as a function of the frequency for different values of the number of the rings $N$ are reported in Fig. 9. From these graphs, the reduction of the resonant frequency from $2.05 \mathrm{GHz}$ in the case of the regular SRRs, down to $1.40 \mathrm{GHz}$ in the case of MSRRs with $N=7$ rings is well evident. We do not show in the graphs of Fig. 9 the results obtained for greater numbers $N$ of the rings because the results are not distinguishable from those ones obtained in the case of seven rings. The aforementioned saturation effect, in fact, takes place and there is no improvement to further increase the number of the inner rings. The higher the number of the rings is, in fact, the lower the increasing rate of the distributed capacitance (the total perimeter of the additional inner rings is smaller and smaller) is. On the other hand, the higher the number of the rings, the smaller the area of the resonators we use to calculate the flux of the magnetic field. This leads to a saturation also in the value of the inductance. The saturation in the values of the capacitance 


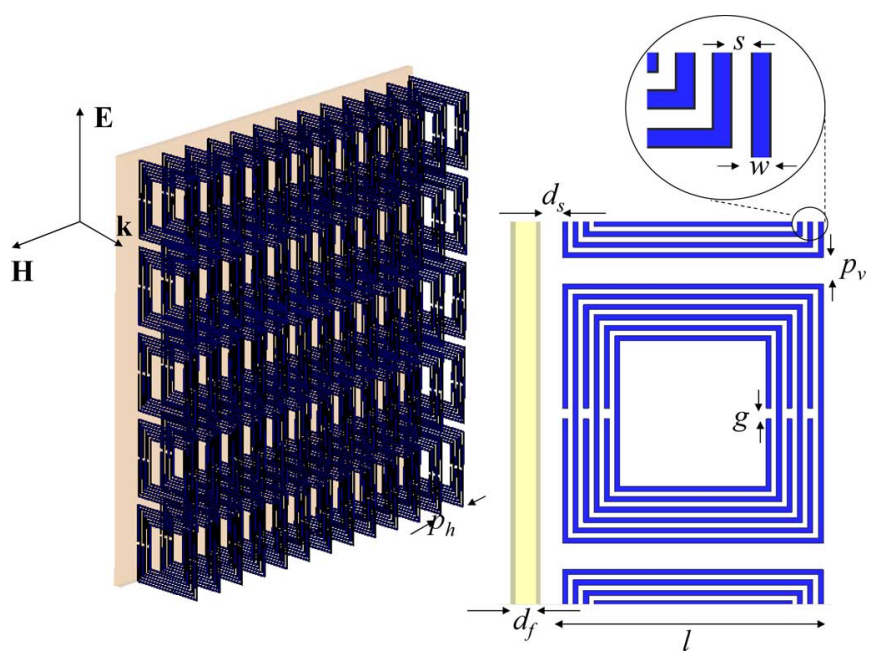

Fig. 8. Geometrical sketch of a microwave absorber based on MSRR resonant magnetic inclusions. The absorber is made by a resistive film of thickness $d_{f}$ and a planar array of MSRRs spaced $d_{s}$ from the resistive film.

and the inductance leads straightforwardly to the saturation of the resonant frequency.

However, in the best case reported in Fig. $9(N=7)$, a considerable reduction of the resonant frequency is obtained with respect to the SRR case. The side length of the MSRR is again $5 \mathrm{~mm}$, but this time the resonant frequency is only $1.40 \mathrm{GHz}$. This means that the inclusion is of the order of $\lambda_{0} / 40$ and that the total thickness of the absorber is around $\lambda_{0} / 36$.

The distribution of the electric-field amplitude, the robustness to the variation of the load impedance, and the performance against the variation of the angle of incidence are not reported here, since they do not differ substantially from what already reported in Figs. 5-7 for the case of SRR-based absorbers.

\section{RESONANT ABSORBERs BASED ON SRS}

A further reduction of the resonant frequency and, thus, of the electrical thickness of the absorbers may be obtained by using SRs [24]-[30]. According to the analytical formulation proposed in [24] and [25], under the assumption that all the mutual interactions between nonadjacent turns are negligible, such a resonator may be modeled in terms of a parallel $L C$ equivalent circuit by using the following expressions for the total capacitance and the total inductance:

$$
\begin{aligned}
C_{\mathrm{SR}} & =C_{0} \frac{l}{4(w+s)} \frac{N^{2}}{N^{2}+1} \sum_{n=1}^{N-1}\left[l-\left(n-\frac{1}{2}\right)(w+s)\right] \\
L_{\mathrm{SR}} & =\frac{\mu_{0}}{2 \pi} l_{\mathrm{avg}}^{\mathrm{SR}}\left[\frac{1}{2}+\ln \left(\frac{l_{\mathrm{avg}}^{\mathrm{SR}}}{2 w}\right)\right]
\end{aligned}
$$

where

$$
l_{\mathrm{avg}}^{\mathrm{SR}}=\frac{4 l N-[2 N(1+N)-3](s+w)}{N}
$$

with $l$ being this time the side length of the outer turn of the spiral, $s$ the separation between two adjacent turns, $N$ the number of

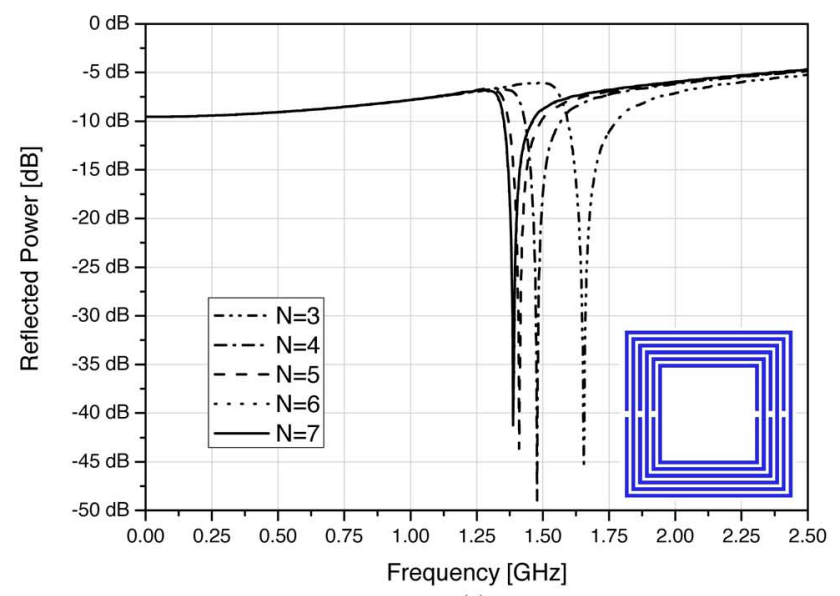

(a)

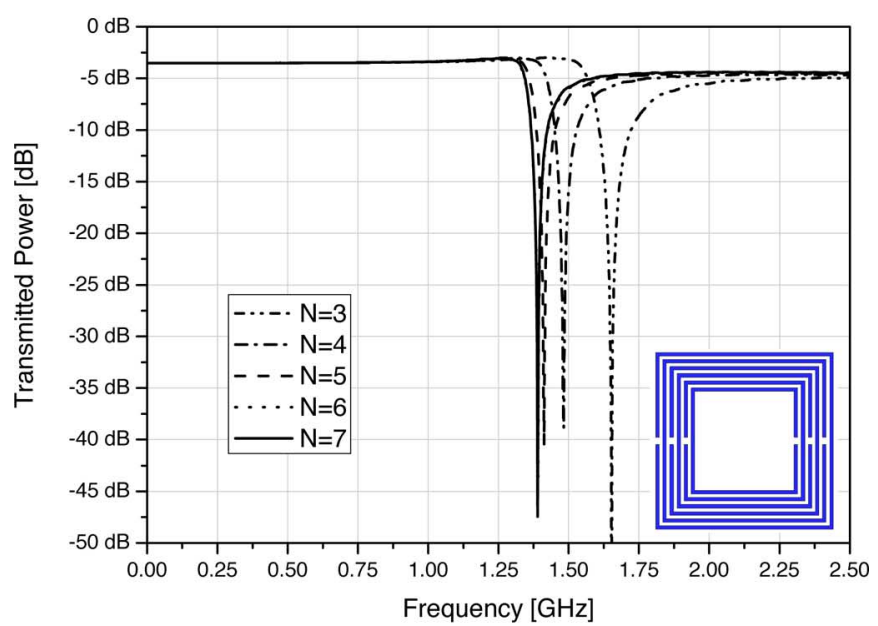

(b)

Fig. 9. Reflected and transmitted power from and through the absorber depicted in Fig. 8 for different values of the number of the rings $N$. The geometrical dimensions of the absorber are: $l=5 \mathrm{~mm}, w=0.1 \mathrm{~mm}, s=0.1 \mathrm{~mm}, g=0.1 \mathrm{~mm}$, $p_{h}=2 \mathrm{~mm}, p_{v}=0.5 \mathrm{~mm}, d_{f}=0.5 \mathrm{~mm}$, and $d_{s}=0.5 \mathrm{~mm}$. The total resistance of the resistive sheet is $377 \Omega$.

the turns, and $w$ again the width of the strip. Similarly to the MSRR, also in the case of the SR, once all the geometrical parameters are known, (5) and (6) may be used to determine the resonant frequency of the SR as the function of the number $N$ of the turns. In parallel to what happens for the MSRR, also for the $\mathrm{SR}$, it is found that, increasing $N$ beyond a certain threshold, the resonant frequency does not reduce any more and a saturation effect takes place [24], [25]. All the details on the physical phenomena related to the SR and to the comparisons with other existing analytical formulations could be found in [24] and [25].

The SRs are used here in a planar-array configuration to obtain an ever more miniaturized resonant absorber. The structure under analysis is the one reported in Fig. 10. In order to verify the reduction of the resonant frequency by using SRs, instead of the regular SRRs and the MSRRs, the simulations are performed keeping unchanged all the geometrical parameters reported in the captions of Figs. 4 and 9.

The obtained results for the reflected and transmitted powers as a function of the frequency for different values of the number 




Fig. 10. Geometrical sketch of a microwave absorber based on SR resonant magnetic inclusions. The absorber is made by a resistive film of thickness $d_{f}$ and a planar array of SRs spaced $d_{s}$ from the resistive film.

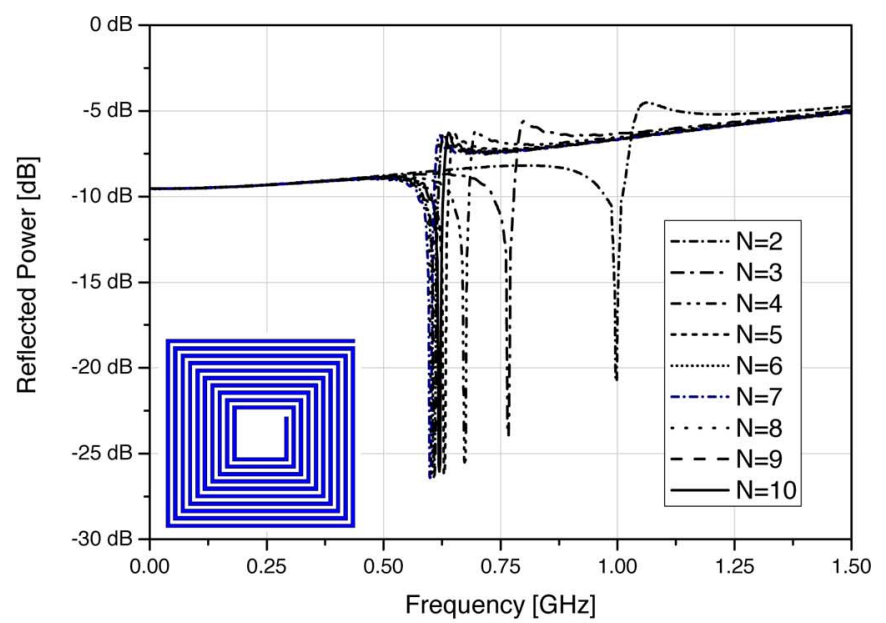

(a)

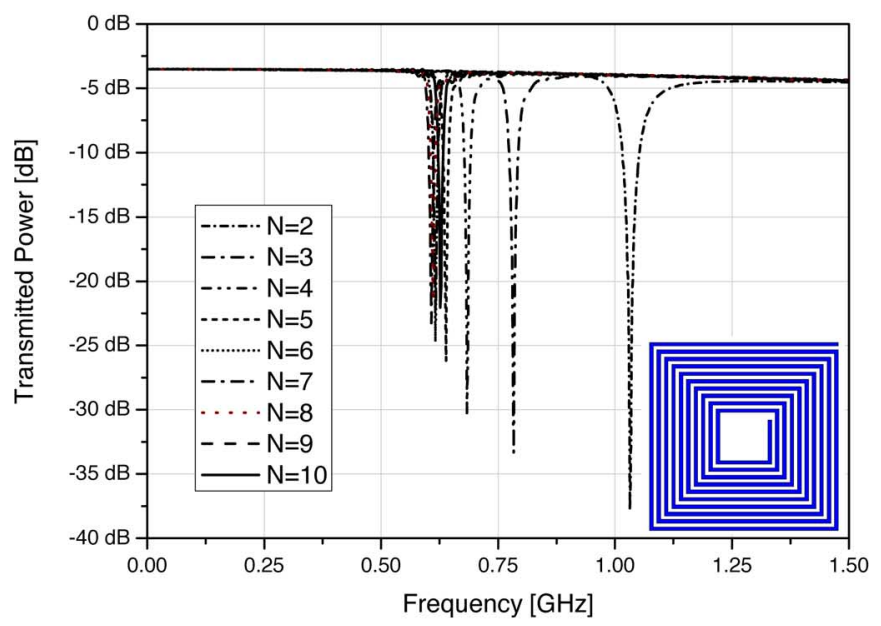

(b)

Fig. 11. Reflected and transmitted power from and through the absorber depicted in Fig. 10 for different values of the number of the turns $N$. The geometrical dimensions of the absorber are: $l=5 \mathrm{~mm}, w=0.1 \mathrm{~mm}, s=0.1 \mathrm{~mm}$, $p_{h}=2 \mathrm{~mm}, p_{v}=0.5 \mathrm{~mm}, d_{f}=0.5 \mathrm{~mm}$, and $d_{s}=0.5 \mathrm{~mm}$. The total resistance of the resistive sheet is $377 \Omega$.

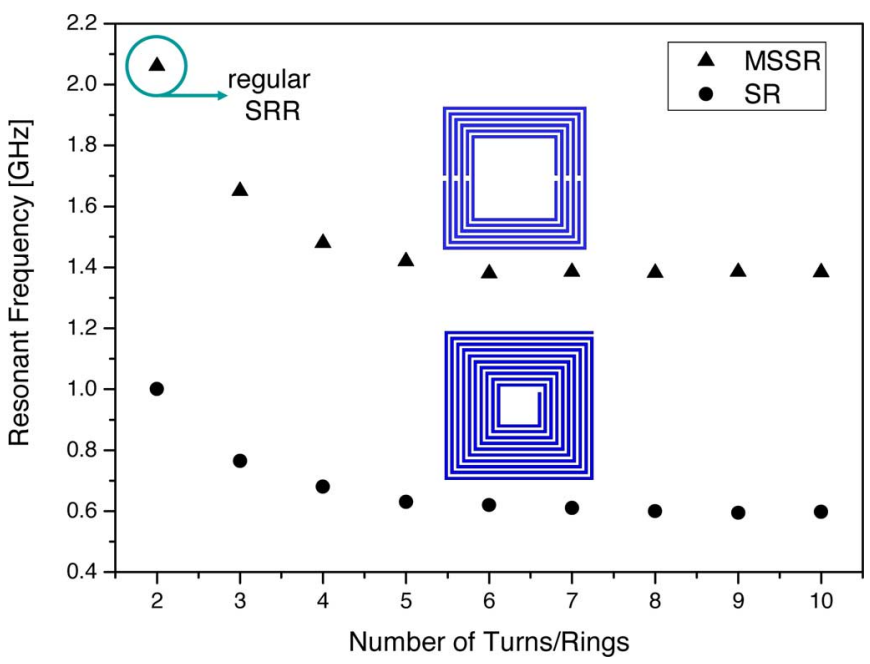

Fig. 12. Comparison of the resonant frequency of the SRR, MSRR, and SR absorbers for different numbers of rings or turns. The geometrical dimensions used in the three cases are all the same $(l=5 \mathrm{~mm}, w=0.1 \mathrm{~mm}, s=0.1 \mathrm{~mm}$, $\left.g=0.1 \mathrm{~mm}, p_{h}=2 \mathrm{~mm}, p_{v}=0.5 \mathrm{~mm}, d_{f}=0.5 \mathrm{~mm}, d_{s}=0.5 \mathrm{~mm}\right)$ and the total resistance of the resistive sheet is set in all cases to $377 \Omega$.

of the turns $N$ of the spiral are reported in Fig. 11. From these diagrams, we observe the reduction of the resonant frequency from $1.40 \mathrm{GHz}$ in the best case of MSRRs with $N=7$ rings, up to $600 \mathrm{MHz}$ in the case of SRs with $N=7$ turns. In contrast with the results presented in Fig. 9, we have shown here the results obtained for greater numbers of turns $(N>7)$ to show how, starting from a certain value of $N$ (in this case $N=7$ ), the resonant frequency of the SR oscillates around a certain value and no further reduction is achieved.

The best result, obtained in the case of $N=7$, shows that a relevant reduction of the resonant frequency is obtained with respect to both the SRR and the MSRR cases. The length of the SR is kept to the value $l=5 \mathrm{~mm}$, but this time the resonant frequency is only $600 \mathrm{MHz}$. This means that the inclusion is of the order of $\lambda_{0} / 100$ and that the total thickness of the absorber is around $\lambda_{0} / 84$.

Further reductions, however, may be obtained by printing the inclusions on a regular dielectric slab with a given dielectric constant. As shown in Section II, in fact, the permeability of the metamaterial slab in the ideal case does not affect the behavior of thin absorbers based on resonant-magnetic inclusions. Therefore, the only effect of the dielectric slab would be to further reduce the resonant frequency of the inclusions, reducing, thereby, the dimensions of the SRs beyond the $\lambda_{0} / 100$ value now presented.

Also in the case of SR-based absorbers, the distribution of the amplitude of the electric field and the performance with the variation of the load impedance and of the incidence angle are not shown here, since they do not differ from what is already presented in Figs. 5-7 for the case of SRR-based absorbers.

In order to summarize all the comparisons made so far between absorbers based on SRR, MSRR, and SR inclusions; in Fig. 12, we present a graph showing the variation of the operating frequencies of absorbers as a function of the number of 
the rings and turns. Note that the SRR may be considered as a particular case of the MSRR with two rings.

\section{CONCLUSION}

A new idea for miniaturized resonant absorbers based on magnetic inclusions has been presented in this paper. A planar array of resonating-magnetic inclusions with a resistive sheet placed in close proximity has shown to effectively absorb most of the impinging power, provided that the resonant inclusions are properly excited. The two main advantages of the absorber concept presented here are: 1) the reduced thickness (close to $\lambda_{0} / 100$ and even beyond) and 2) the absence of a metallic plate on the back of the absorbing structure. Both these aspects have been discussed in the paper through proper numerical simulations.

Different kinds of air-immersed magnetic inclusions, namely SRRs, MSRRs, and SRs, have been considered to design absorbers. The employment of regular SRRs has shown to lead an absorber-electrical thickness of the order of $\lambda_{0} / 20$. A reduction of the electrical thickness up to approximately $\lambda_{0} / 40$ may be obtained using MSRRs, while further reductions of the electrical thickness close to $\lambda_{0} / 100$ may be easily obtained through the employment of the SRs. Further reductions are envisaged by printing the inclusions on dielectric slabs with given permittivities. It is worth noting that all the geometrical dimensions used in the paper (width of the strips, separation between the rings of the MSRRs, or the turns of the SRs, gaps in the rings, etc.) are not extreme and, indeed, can be easily obtained through well established and relatively cheap fabrication techniques at microwaves.

Furthermore, we remark here that all the simulations have been conducted without considering realistic losses in the metals. Nevertheless, the absorber represents a unique component, where losses do not limit the applicability of metamaterials in practical designs and, indeed, may help to improve the performance of the components.

As a final remark, we notice that the proposed layouts, working for one single polarization, can be easily extended to work for double polarization operation (which is usually the case of radar applications), exploiting more complicated unit cells for isotropic-metamaterial designs, as the ones proposed, for instance in [31].

\section{REFERENCES}

[1] H. Tao, N. I. Landy, C. M. Bingham, X. Zhang, R. D. Averitt, and W. J. Padilla, "A metamaterial absorber for the terahertz regime: Design, fabrication and characterization," Opt. Exp., vol. 16, no. 10, pp. 71817188,2008

[2] N.I. Landy, S. Sajuyigbe, J. J. Mock, D. R. Smith, and W. J. Padilla, "Perfect metamaterial absorber," Phys. Rev. Lett., vol. 100, pp. 2074021-207402-4, 2008.

[3] Y. Aritzour, Y. A. Urzhumov, and G. Shvets, "Wide-angle infrared absorber based on a negative-index plasmonic metamaterial," Phys. Rev. B, vol. 79, pp. 045131-1-045131-5, 2009.

[4] B. Wang, T. Koschny, and C. M. Soukoulis, "Wide-angle and polarizationindependent chiral metamaterial absorber," Phys. Rev. B, vol. 80, pp. 033108-1-033108-4, 2009.
[5] W. W. Salisbury, "Absorber body for electromagnetic waves," U.S. Patent 2599 944, Jun. 10, 1952

[6] R. L. Fante and M. T. McCormack, "Reflection properties of the Salisbury screen," IEEE Trans. Antennas Propag., vol. AP-30, no. 10, pp. 14431454, Oct. 1968.

[7] D. J. Kern and D. H. Werner, "A genetic algorithm approach to the design of ultra-thin electromagnetic bandgap absorbers," Microw. Opt. Technol. Lett., vol. 38, no. 1, pp. 61-64, May 9, 2003.

[8] H. Mosallaei and K. Sarabandi, "A one-layer ultra-thin meta-surface absorber," in Proc. IEEE Antennas Propag. Soc. Int. Symp., Jul. 3-8, 2005, vol. 1B, pp. 615-618.

[9] S. Cui, D. S. Weile, and J. L. Volakis, "Novel planar electromagnetic absorber designs using genetic algorithms," IEEE Trans. Antennas Propag., vol. AP-54, no. 6, pp. 1811-1817, Jun. 2006.

[10] C. R. Simovski, M. V. Ermutlu, A. A. Sochava, and S. A. Tretyakov, "Magnetic properties of novel high-impedance surfaces," IET Microw. Antennas Propag., vol. 154, pp. 1-8, 2007.

[11] R. W. Ziolkowski and N. Engheta, (Guest Eds.), "Special Issue on Metamaterials," IEEE Trans. Antennas Propag., vol. AP-51, no. 10, Oct. 2003.

[12] A. Ishimaru, L. Seung-Woo, Y. Kuga, and V. Jandhyala, "Generalized constitutive relations for metamaterials based on the quasi-static Lorentz theory," IEEE Trans. Antennas Propag., vol. AP-51, no. 10, pp. 25502557, Oct. 2003.

[13] F. Bilotti, "Application of metamaterials for miniaturized components," presented at the Metamaterials Ind., Short Course Ind. SMEs, Jouy-enJosas, France, Nov. 28-30, 2005.

[14] F. Bilotti, A. Alù, N. Engheta, and L. Vegni, "Metamaterial sub-wavelength absorbers," presented at the Nanosci. Nanotechnol. Symp., Frascati, Italy, Nov. 14-16, 2005.

[15] F. Bilotti, A. Alù, N. Engheta, and L. Vegni, "Features of a metamaterial based microwave absorber," in Proc. Third Workshop Metamaterials Spec. Mater. Electromagn. Appl. TLC, Rome, Italy, Mar. 30-31,2006, p. 60.

[16] F. Bilotti, A. Alù, N. Engheta, and L. Vegni, "Compact microwave absorbers utilizing single negative metamaterial layers," in Proc. IEEE AP-S Int. Symp. USNC/URSI Nat. Radio Sci. Meeting, CD Digest, Albuquerque, Jul. 9-14, 2006, p. 152

[17] S. I. Maslovski, S. A. Tretyakov, and P. A. Belov, "Wire media with negative effective permittivity: a quasi-static model," Microw. Opt. Technol. Lett., vol. 35, no. 1, pp. 47-51, 2002.

[18] J. B. Pendry, A. J. Holden, D. J. Robbins, and W. J. Stewart, "Magnetism from conductors and enhanced nonlinear phenomena," IEEE Trans. Microw. Theory Tech., vol. MTT-47, no. 11, pp. 2075-2084, Nov. 1999.

[19] F. Bilotti, L. Nucci, and L. Vegni, "An SRR based microwave absorber," Microw. Opt. Technol. Lett., vol. 48, no. 11, pp. 2171-2175, Nov. 2006.

[20] C. R. Simovski, M. S. Kondratiev, and S. He, "Array of C-shaped wire elements for extreme reduction of Dallenbach low-reflecting shields," $J$. Electromag. Waves Appl., vol. 14, pp. 1335-1352, 2000.

[21] C. R. Simovski, M. S. Kondratiev, and S. He, "Array of C-shaped wire elements for the reduction of reflection from a conducting plane," Microw. Opt. Technol. Lett., vol. 25, pp. 302-307, 2000.

[22] CST Microwave Studio ${ }^{\mathrm{TM}}$ 5.0, Inc., [Online]. Available: www.cst.com

[23] J. Gordon, C. L. Holloway, and A. Dienstfrey, "A physical explanation of angle independent reflection and transmission properties of metafilm/metasurfaces," IEEE Antennas Propag. Wireless Lett., vol. 8 , pp. 1127-1130, 2009.

[24] F. Bilotti, A. Toscano, and L. Vegni, "Design of spiral and multiple splitring resonators for the realization of miniaturized metamaterial samples," IEEE Trans. Antennas Propag., vol. 55, no. 8, pp. 2258-2267, Aug. 2007.

[25] F. Bilotti, A. Toscano, L. Vegni, K. B. Alici, K. Aydin, and E. Ozbay, "Equivalent circuit models for the design of metamaterials based on artificial magnetic inclusions," IEEE Trans. Microw. Theory Tech., vol. MTT55, no. 12, pp. 2865-2873, Dec. 2007.

[26] J. D. Baena, J. Bonache, F. Martín, R. Marqués, F. Falcone, T. Lopetegi, M. A. G. Laso, J. García-García, M. F. Portillo, and M. Sorolla, "Equivalent-circuit models for split-ring resonators and complementary split-ring resonators coupled to planar transmission lines," IEEE Trans. Microw. Theory Tech., vol. MTT-53, no. 4, pp. 1451-1461, Apr. 2005.

[27] J. D. Baena, R. Marques, F. Medina, and J. Martel, "Artificial magnetic metamaterial design by using spiral resonators," Phys. Rev. B, vol. 69, pp. 014402-1-014402-5, 2004

[28] K. Buell, H. Mosallaei, and K. Sarabandi, "A substrate for small patch antennas providing tunable miniaturization factors," IEEE Trans. Microw. Theory Tech., vol. MTT-54, no. 1, pp. 135-146, Jan. 2006.

[29] K. B. Alici, F. Bilotti, L. Vegni, and E. Ozbay, "Optimization and tunability of deep subwavelength resonators for metamaterial applications: 
Complete enhanced transmission through a subwavelength aperture," Opt. Exp., vol. 17, pp. 5933-5943, 2009.

[30] K. B. Alici, F. Bilotti, L. Vegni, and E. Ozbay, "Miniaturized negative permeability materials," Appl. Phys. Lett., vol. 91, pp. 071121-1-0711213, 2007.

[31] J. D. Baena, L. Jelinek, and R. Marques, "Electrically small isotropic three-dimensional magnetic resonators for metamaterial design," Appl. Phys. Lett., vol. 88, pp. 134108-1-134108-3, 2006.

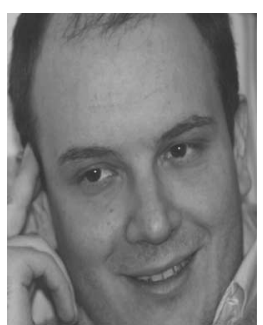

Filiberto Bilotti (S'97-M'03-SM'06) was born in Rome, Italy, on April 25, 1974. He received the Laurea (summa cum laude) and Ph.D. degrees in electronic engineering from the University of Roma Tre, Rome, Italy, in 1998 and 2002, respectively.

Since 2002, he has been with the Department of Applied Electronics, University of Roma Tre, where he is currently an Assistant Professor of electromagnetic field theory. His research interests include the microwave, THz, and optical applications of complex media, metamaterials and metasurfaces, the analysis and synthesis of planar and conformal integrated components and phased antenna arrays, and the development of improved numerical algorithms for an efficient analysis of printed antennas and circuits. He is the author of more than 300 papers on international journals, conference proceedings, and book chapters. Since 1999, he has been a National Expert of the European actions on antenna technology and design COST260, COST284, and COST "Assist." Since 2007, he has also been an Expert Member of COST MP0702: "Towards Functional Sub-Wavelength Photonic Structures.” Since 2003, he has been a Technical Reviewer of the European Commission for scientific projects in the fields of metamaterials and antennas.

Dr. Bilotti is a member of the Steering Committee of the European Doctoral School on Metamaterials and the Organizer of several international school events and international workshops and conferences in the field of metamaterials. He has been the local Organizer of the First Congress on Advanced Electromagnetic Materials and Metamaterials in Microwaves and Optics-Metamaterials, Rome, Italy, October 2007 and the Chairman of the Steering Committee of Metamaterials, Pamplona, Spain, September 2008; Metamaterials, London, U.K., September 2009; and Metamaterials, Karlsruhe, Germany, September 2010. He was a member of the Technical Program, the Steering Committee, and the Organizing Committee of several national and international conferences and training events related to metamaterials, as an Organizer and the Chairman of special sessions focused on the applications of metamaterials at microwave and optical frequencies, as a member of the Editorial Board of the journals: Metamaterials and International Journal on RF and Microwave Computer-Aided Engineering, and as a Technical Reviewer of the major international journals related to electromagnetic field theory and metamaterials. From 2004 to 2008, he was a member of the governing bodies of METAMORPHOSE, the European Network of Excellence on Metamaterials, where he was the Coordinator of spreading activities. Since 2007, he has been the member of the Board of Directors of the Virtual Institute for Artificial Electromagnetic Materials and Metamaterials (the European Society on Metamaterials), where he is the Coordinator of the educational activities. He is a member of the Optical Society of America and the International Society for Optical Engineers. He was the recipient of the Raj Mittra Travel Grant Senior Researcher Award in 2007.

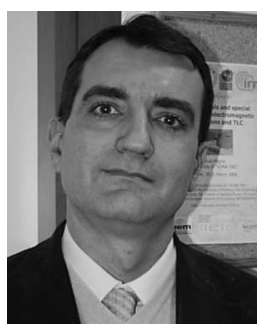

Alessandro Toscano was born in Capua, Italy, on June 26, 1964. He received the Laurea and the Ph.D. degrees, both in electronic engineering, from the University "La Sapienza," Rome, Italy, in 1988 and 1993, respectively.

From 1994 to 2002, he was an Assistant Professor of electromagnetic field theory at the Department of Electronic Engineering, University of Roma Tre. Since 2002, he has been an Associate Professor in the Department of Applied Electronics, University of Roma Tre, where he is currently involved in teaching, research, and academic activities. He has been the Advisor or Coadvisor of more than 50 theses, some of which have received national and international recognitions and awards. Since 2004, he has been involved in the teaching activities for the Ph.D. Program in electronics and telecommunications at the University of Roma Tre and for Master's Programs in telecommunications, complex electronic systems, and electronic defence organized by the Headquarter of the Italian Military Forces in cooperation with the University of Roma Tre. Since 1990 he has been participating as a National Expert Member for Italy at the European Actions COST (Coopération européenne dans le domaine de la recherche Scientifique et Technique): COST260 on "Smart Antennas: Computer Aided Design and Technology" and COST284 on "Innovative Antennas for Emerging Terrestrial and Space-based Applications," and attending the most important international conferences in the electrical engineering field, as the Presenter and the coauthor of regular contributions, key-note speaker, invited lecturer, session chairman, and session organizer. He is the Reviewer of the major international journals related to electromagnetic field theory, material sciences, antennas and propagation, microwave and optical devices. He has been actively involved in several national, European, and international research projects/contracts, as engineer in charge of the project/contract, team leader, task supervisor, and regular member performing part of the research project/contract. He is the coauthor of more than 200 peer reviewed scientific papers on international journals, review papers, conference proceedings, book chapters, books.

Dr. Toscano is currently a member of the scientific/steering/technical program/organizing committees of several conferences, workshops, schools, and seminars concerning metamaterials and electromagnetic compatibility. He was the local Cochair of "Metamaterials 2007-The First International Congress on Advanced Electromagnetic Materials in Microwaves and Optics."

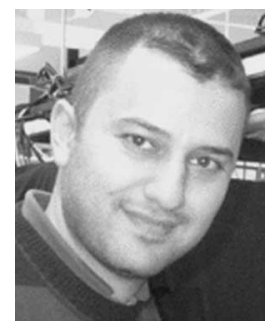

Kamil Boratay Alici was born in Sivas, Turkey, on January 12, 1981. He received the B.S. degree in physics from the Bilkent University, Ankara, Turkey, in 2004. He is currently working toward the Ph.D. degree at the Nanotechnology Research Center, Bilkent University.

His current research interests include photonic and electromagnetic metamaterials and their applications such as superlensing, enhanced transmission, millimeter-wave negative-index materials, miniaturized absorbers, electrically small antennas and negative refraction in photonic crystals. He is the author of 16 papers and coauthor of 6 papers in scientific journals.

Mr. Alici was the recipient of the Undergraduate Scholarship (2001-2004) and Graduate Scholarship (2004-2009) of the Scientific and Technological Research Council of Turkey.

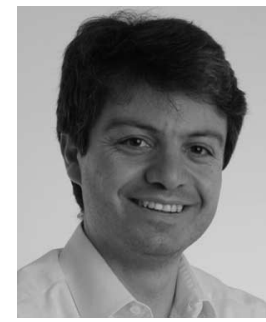

Ekmel Ozbay received the B.S. degree in electrical engineering from the Middle East Technical University, Ankara, Turkey, in 1983,the M.S. and $\mathrm{Ph} . \mathrm{D}$. degrees in electrical engineering from the Stanford University, Stanford, CA, in 1989 and 1992, respectively.

From 1992 to 1994, he was a Scientist in DOE, Ames National Laboratory, Iowa State University in the area of photonic band gap materials. In 1995, he joined Bilkent University, Ankara, Turkey, where he is currently a Full Professor in electrical and electronics engineering department and physics department. He is also the Director of Nanotechnology Research Center, Bilkent University, where he has been involved in nanophotonics, metamaterials, metal organic chemical vapor deposition growth and fabrication of $\mathrm{GaN}$-based electronic and photonic devices, photonic crystals, and high-speed optoelectronics. He is the author or coauthor of $235 \mathrm{SCI}$ journal papers, and these papers have received more than $5500 \mathrm{SCI}$ citations. He was a topical editor for Optics Letters during 2002-2008. Since 2006, he has been an Editor for Photonics and Nanostructures: Fundamental Applications.

Prof. Ozbay was the recipient of the 1997 Adolph Lomb Medal of Optical Society of America and 2005 European Union Descartes Science Award. 




Lucio Vegni (M'73) was born in Castiglion Fiorentino, Italy, on June 20, 1943. He received the Electronic Engineering degree from the University of Rome, Rome, Italy.

Following a period of work at Standard Elektrik Lorenz in Stuttgart, Germany, as an Antenna Designer, he joined Istituto di Elettronica of the University of Rome, where he was a Researcher in applied electronics. From 1976 to 1980, he was Researcher Professor of applied electronics at the University of L'Aquila. From 1980 to 1985 , he was a Researcher Professor of applied electronics, and from 1985 to 1992, he was an Associate Professor of electromagnetic compatibility at the University of Rome "La Sapienza," Since 1992, he has been with the University of Roma Tre, Rome, Italy, where he is currently a Full Professor of electromagnetic field theory, the President of the undergraduate and graduate Courses in electronic engineering, the President of the Doctoral Courses in electromagnetisms, telecommunications, and bio-engineering, the Head of the Applied Electromagnetic Laboratory. His research interests include microwave and millimeter-wave circuits and antennas with particular emphasis to the EMC problems and in the field of metamaterials. Specifically, he was active in studies of partial coherence radio links, with particular attention on multipath electromagnetic propagation effects until
1977. He, then, moved to the area of integrated microwave circuits, where he studied the electromagnetic modeling of microstrip planar circuits and antennas. In cooperation with industry, he was engaged in the development of integrated microstrip antennas for satellite applications and in study of radiating system electromagnetic compatibility problems from 1985 to 1990 . Since 1990 , he has been actively involved with theoretical and numerical aspects of new planar antennas modeling involving unconventional materials. In these recent studies, he offered new contributions to equivalent circuit representations of planarmicrowave components and new variational formulations for their numerical simulations. Finally, in the area of unconventional materials, he has given noteworthy contributions to the study of chiral and omega grounded dielectric slab antennas. He has authored or coauthored more than 500 international papers appearing in journals, transactions, and conferences.

Prof. Vegni has been the Organizer and the Chairman of the second and third edition of the "International Workshop on Metamaterials and special materials for electromagnetic applications and TLC," Rome, Italy, in 2004 and 2006, respectively. He has been also the local Chairman of "Metamaterials 2007The First International Congress on Advanced Electromagnetic Materials in Microwaves and Optics," Rome, Italy, in 2007. He is a member of the European Chiral Group, the Network of Excellence METAMORPHOSE, and the Italian Electrical and Electronic Society. 